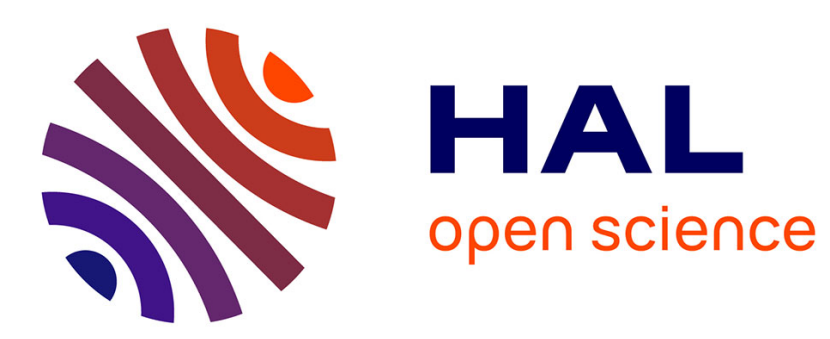

\title{
A new interpolation technique to deal with fluid-porous media interfaces for topology optimization of heat transfer
}

Delphine Ramalingom, Pierre-Henri Cocquet, Alain Bastide

\section{- To cite this version:}

Delphine Ramalingom, Pierre-Henri Cocquet, Alain Bastide. A new interpolation technique to deal with fluid-porous media interfaces for topology optimization of heat transfer. Computers and Fluids, 2018, 10.1016/j.compfluid.2018.04.005 . hal-01618684

\section{HAL Id: hal-01618684 \\ https://hal.univ-reunion.fr/hal-01618684}

Submitted on 18 Oct 2017

HAL is a multi-disciplinary open access archive for the deposit and dissemination of scientific research documents, whether they are published or not. The documents may come from teaching and research institutions in France or abroad, or from public or private research centers.
L'archive ouverte pluridisciplinaire HAL, est destinée au dépôt et à la diffusion de documents scientifiques de niveau recherche, publiés ou non, émanant des établissements d'enseignement et de recherche français ou étrangers, des laboratoires publics ou privés. 


\title{
A new interpolation technique to deal with fluid-porous media interfaces for topology optimization of heat transfer
}

\author{
Delphine Ramalingom ${ }^{\mathrm{a}, *}$, Pierre-Henri Cocquet $^{\mathrm{a}}$, Alain Bastide ${ }^{\mathrm{a}}$ \\ ${ }^{a}$ Université de La Réunion, Laboratoire PIMENT \\ 117 Avenue du Général Ailleret \\ 97430 Le Tampon, France
}

\begin{abstract}
This paper proposes a new interpolation technique based on density approach to solve topology optimization problems for heat transfer. Problems are modeled under the assumptions of steady-state laminar flow using the incompressible Navier-Stokes equations coupled to the convection-diffusion equation through the Boussinesq approximation. The governing equations are discretized using finite volume elements and topology optimization is performed using adjoint sensitivity analysis. Material distribution and effective conductivity are interpolated by two sigmoid functions respectively $h_{\tau}(\alpha)$ and $k_{\tau}(\alpha)$ in order to provide a continuous transition between the solid and the fluid domains. Comparison with standard interpolation function of the literature (RAMP function) shows a smaller transition zone between the fluid and the solid thereby, avoiding some regularization techniques. In order to validate the new method, numerical applications are investigated on some cases from the literature, namely the single pipe and the bend pipe. Lastly, as
\end{abstract}

\footnotetext{
*Corresponding author: delphine.ramalingom@univ-reunion.fr
} 
two new parameters are introduced thanks to the interpolation functions, we study their impact on results of the optimization problem. The study shows that the proposed technique is a viable approach for designing geometries and fluid-porous media interfaces are well-defined.

Keywords: Topology optimization, Heat transfer, Interpolation function, Sigmoid function, Continuous adjoint method, Finite volume method

\section{1. Introduction}

2 Since its introduction by Bendsoe and Sigmund [1] for solid mechanics 3 problems, topology optimization has become a powerful and increasingly 4 popular tool for designers and engineers for design process. Topology opti5 mization is a material distribution method for finding the optimal structure, 6 for a given problem subject to design constraints. Contrarily to shape op7 timization where the topology (i.e. the number of boundaries and connec8 tivity) is predetermined, topology optimization allows introduction of new boundaries during the design process.

Topology optimization was pioneered for Stokes flow by Borrvall and Petersson [4]. They introduced a friction term yielding the generalized Stokes equations. Gersborg-Hansen [9] and Olesen et al. [8] extended topology optimization for fluid flow problems to the Navier-Stokes equations.

In topology optimization, the material distribution is parametrized by defining a design variable $\alpha \in\{0 ; 1\}$. This variable is discrete and should either represent solid material $(\alpha=1)$ or fluid $(\alpha=0)$. A common approach to solve the topology optimization problem with this discrete value as optimization parameter, is to change it into a continuous one by introducing a 
porous media with a continuous permeability variable for each element. This method, known as the Brinkman penalization, leads to a problem where flow and (almost) non-flow regions are developed by allowing interpolation between the lower and upper value of permeability. Generally, authors used the density interpolation function proposed by Borrvall and Petersson [4] or a reformulated version of their convex and $q$-parametrized interpolation function. The parameter $q>0$ is a penalty parameter that is used to control the level of 'gray' in the optimal design. However, authors had also experienced problems with locally optimal solutions. Therefore, they considered a two-steps solution procedure where the problem was first solved with a small penalty value of $q=0.01$ for example and then the result is used as initial case for the problem with a penalty value of $q=0.1[4,8]$ or $q=1$ [15]. The mathematical foundation of the interpolation of $\alpha$ was further investigated by Evgrafov [14] where the limiting cases of pure fluid and solid were included. Brinkman approach has since been used for several problems as transport problem [28], reactive [32] and transient flows [33, 3], fluid-structure interaction [36] and also flows driven by body forces [37].

A variation of the approach is presented by Guest and Prevost [2]. They proposed to regularize the solid-fluid structure by treating the material phase as a porous medium where fluid flow is governed by Darcy's law. In their approach, flows through voids are governed by Stokes flow and, when the solid phase is impermeable, discrete no-slip condition is simulated by assigning a low permeability to the solid phase. There exists other alternatives to Brinkman penalization in the literature. The level set approach to topology optimization has been applied to fluid flows problems [34, 46, 41], and 
recently the level set approach was combined with the extended finite element method (XFEM) by Kreissl and Maute [5] and by Jenkins et al. [40]. The main drawback of the level set appraoch is the constraint of remeshing throughout the optimization process.

The second difficulty in topology optimization is to deal with the difference in thermal conductivity in the solid and fluid domains. Most publications interpolate the conductivity using the SIMP method (Solid Isotropic Material with penalization). This method allows to deal with the discrete nature of conductivity material distribution. So, authors $[6,15,30,36]$ considered a continuous local thermal conductivity controlled by the design parameter $\alpha$ ranging from 0 to 1 . Thanks to this function, the optimization algorithm is able to reallocate thermal conductivity material, or creating holes in its structure to reach the objective function. Moreover, the convex and $p$-parametrized function interpolation is similar to the density interpolation function of Borrvall and Petersson [4] or the RAMP (Rational Approximation of Material Properties)-style function as introduced by Stolpe [16, 30, 7] . Other methods have also been investigated. Matsumori et al. [19] presented results with a linear-interpolated design-dependant volumetric heat generation. Dede [20] used a linear interpolation for thermal conductivity. Thus, the main issue is to deal with intermediate design variables and nonphysical flow solutions.

There are three main categories of algorithms to solve topology optimization problems : gradient-free, gradient-based and hessian-based algorithms. In topology optimization problems with large number of design variables, gradient-based algorithms are used to find accurate solutions efficiently. One 
of the advantages of the interpolation functions described above is the possibility of using gradient-based continuous optimization methods. These methods are based on derivatives in order to find extrema, and are the so-called sensitivity analysis. It aims at evaluating the derivative of objective function with respect of $\alpha$. Gradient-based algorithm is widely used by several authors $[27,21,30,35,47]$. Moreover, since most of the topology optimization problems involve a huge number of design variable, specific gradient-based optimization algorithms must be chosen to handle this difficulty. A famous algorithm from the literature is the MMA (Method of Moving Asymptotes) developed initially by Svanberg [43]. In order to reduce the computational costs, adjoint approach consisting to calculate the sensitivities of the objective function by an adjoint state has been adopted. Other methods have been explored to reduce the computation cost: the multigrid preconditioned conjugate gradients (MGCC) by Amir et al. [22], multi-resolution multi-scale topology optimization technique by Kim et al. [23], the technique of using adaptive design variable fields by Guest et al. [26].

Moreover, various regularization techniques based on filtering of either the design variable $\alpha$ or the sensitivity $\frac{\partial f}{\partial \alpha}[1,10,15,30,48]$ exist to ensure well-posed topology optimization problems. The regularization works by defining a certain length scale $r_{0}$ below which any features in $\alpha$ or $\frac{\partial f}{\partial \alpha}$ are smeared out by the filter; that results in optimized structures with a minimal feature size $r_{0}$ independent of the mesh refinement. As mentioned by some authors [15, 29], these regularization techniques allow to avoid checkerboard problems.

This paper proposes a new interpolation technique in order to solve a 
heat transfer topology optimization problem. Design material and effective conductivity are interpolated respectively by a function $h_{\tau}(\alpha)$ and another function $k_{\tau}(\alpha)$ in order to provide a continuous transition between the solid and the fluid domains. These interpolation functions avoid the use of some regularization techniques because the problem can be solved in one-step without a new value of the convexity parameter. Moreover, these interpolation functions allow a smaller transition zone between the fluid and solid regions. To prove this claim and get more qualitative results, the size of these transition zones is explicitly computed and comparison with standard RAMP interpolation is lead. In order to validate the new method, some numerical applications are investigated on the single pipe and the bend pipe cases of the literature. Lastly, as two new parameters are introduced thanks to the interpolation functions, we study their impact on the results of a optimization problem.

\section{Governing equation}

The main goal of this paper is to solve topology optimization problems for heat transfer in fluid flow. The latter can be written in the general form below:

$$
\begin{array}{ll}
\text { Minimize } & \mathcal{J}(\mathbf{u}, p, \theta)=\int_{\Omega} \mathcal{J}_{\Omega}(\mathbf{u}, p, \theta) d \Omega+\int_{\Gamma} \mathcal{J}_{\Gamma}(\mathbf{u}, p, \theta) d \Gamma \\
\text { Subject to } & \text { Governing equation for }(\mathbf{u}, p, \theta) \\
& \text { Boundary conditions on } \Gamma
\end{array}
$$

where $\mathbf{u}, p$ and $\theta$ are respectively the dimensionless velocity, pressure and temperature, $\Omega$ is a bounded open set of $\mathbb{R}^{d}, d=2,3$, with boundary $\Gamma=$ $\partial \Omega$. The function $\mathcal{J}_{\Omega}$ and $\mathcal{J}_{\Gamma}$ are some cost functional modeling a physical 
effect one wish to minimize. We assume that $\Gamma$ can be decomposed as $\Gamma=$ $\Gamma_{1} \cup \Gamma_{2} \cup \Gamma_{\text {in }} \cup \Gamma_{\text {out }}$ where $\Gamma_{\text {in }}$ is the inlet, $\Gamma_{\text {out }}$ the outlet and $\Gamma_{1}, \Gamma_{2}$ are going to be considered as walls.

For the governing equation, the flows considered in this study are assumed Newtonian and incompressible, steady and laminar. The inverse permeability field is introduced in the steady-state Navier-Stokes equations as a source term $h_{\tau}(\alpha) \mathbf{u}$ yielding a Brinkman model with a convection term. The set of dimensionless equations governing the conservation of momentum, mass and energy for incompressible steady-state fluid flow are the following:

$$
\begin{aligned}
& (\mathbf{u} \cdot \nabla) \mathbf{u}=-\nabla p+R e^{-1} \Delta \mathbf{u}-h_{\tau}(\alpha) \mathbf{u}+\operatorname{Ri} \theta \overrightarrow{e_{y}} \quad \text { in } \Omega, \\
& \nabla \cdot \mathbf{u}=0 \quad \text { in } \Omega, \\
& \nabla \cdot(\mathbf{u} \theta)=\operatorname{div}\left(\operatorname{Re}^{-1} \operatorname{Pr}^{-1} k_{\tau}(\alpha) \nabla \theta\right) \quad \text { in } \Omega \text {. }
\end{aligned}
$$

The reduced dimensionless temperature is $\theta=\left(T-T_{0}\right) / \Delta T$ and the uniform heat flux $\Phi=\partial_{n} \theta$ on some part of $\Gamma_{1}$ is equal to $\lambda_{f} \Delta T / l$, with $\lambda_{f}$ the thermal conductivity of the fluid. Parameters governing the flow are the Reynolds number defined as $R e=U_{0} l / \nu$, with $U$ the reference velocity (i.e mean velocity here) and $l$ is the hydraulic diameter, the Richardson number $R i=G r / R e^{2}$ where $G r$ is the Grashof number defined as $G r=g \beta \Delta T l^{3} / \nu^{2}$. $h_{\tau}(\alpha)$ corresponds to the ratio between a kinematic viscosity and a permeability and $\alpha$ is the spatially varying design variable field determined by the optimization algorithm. Regions with low permeability can be considered as solid regions since (at least formally) the velocity of the fluid vanishes in such region, and those with very high permeability regions are interpreted as pure fluid. The interpolation function for the adimensional thermal conductivity is given by $k_{\tau}(\alpha)$. 
We consider the following set of boundary conditions:

$$
\begin{array}{lll}
\mathbf{u}=0, & \partial_{n} \theta=-1 & \text { on } \Gamma_{1}, \\
\mathbf{u}=0, & \partial_{n} \theta=0 & \text { on } \Gamma_{2}, \\
\mathbf{u}_{n}=1, \mathbf{u}_{t}=0 & \theta=0 & \text { on } \Gamma_{\text {in }}, \\
\partial_{n} \mathbf{u}=0, & \partial_{n} \theta=0, \quad p=0 & \text { on } \Gamma_{\text {out }},
\end{array}
$$

where $\mathbf{u}_{n}$ and $\mathbf{u}_{t}$ are the normal and tangential components of primal velocities, respectively. To summarize, we require a constant horizontal velocity and a constant temperature $\theta_{0}$ at the inlet, vanishing gradient for both velocity and temperature of fluid at the outlet. Homogeneous Dirichlet for the velocity and Neumann boundary condition are prescribed for the temperature on the walls. It is worth noting that boundary conditions on the outlet have been considered in [39] and ensure that the fluid does not re-enter in the domain.

\section{Interpolation}

The goal of topology optimization is to end up with binary designs, i.e avoid that the design variables take other value than those representing the fluid or the solid. The most important thing is to be sure that the intermediate regions (the transition zones) are unattractive with respect to the optimization problem. This is usually carried out by penalizing the intermediate densities with respect to the material parameters, such as inverse permeability and effective conductivity. A standard approach is to use some convex interpolation (RAMP) function $[4,30,15]$. In this section, we propose to use another interpolation function hence interpolate the inverse permeability and the effective conductivity using sigmoid functions. After giving the 
177

definition of our interpolation function, we show that the transition zones of the sigmoid are much smaller than those of the standard RAMP function therefore motivating the use of such method in topology optimization.

\subsection{Interpolation of porosity using a sigmoid function}

Inverse permeability is interpolated with a sigmoid function

$$
h_{\tau}\left(\alpha, \alpha_{0}\right)=\alpha_{\max }\left(\frac{1}{1+\exp \left(-\tau\left(\alpha-\alpha_{0}\right)\right)}-\frac{1}{1+\exp \left(\tau \alpha_{0}\right)}\right)
$$

where $\alpha \in\left[0, \alpha_{\max }\right]$ with $\alpha_{\max }$ being the maximal value $h_{\tau}$ can reach. Direct computations show that $h_{\tau}\left(0, \alpha_{0}\right)=0$ and that the following point-wise convergence holds

$$
\lim _{\tau \rightarrow+\infty} h_{\tau}\left(\alpha, \alpha_{0}\right)= \begin{cases}0 & \text { if } \alpha<\alpha_{0}, \\ \alpha_{0} / 2 & \text { if } \alpha=\alpha_{0}, \\ \alpha_{\max } & \text { if } \alpha>\alpha_{0} .\end{cases}
$$

This shows that $h_{\tau}$ is a smooth regularization of a Heaviside step function. From (5), one can see that $\alpha_{0}$ can be tuned to control the size of the fluid part in the computational domain. Finally, note that the definition of our interpolation function has to be changed when $\alpha_{0}=0$ since we require $\alpha \geq 0$ and (4) would lead to a regularization of a step function satisfying $h_{\tau}(0,0)=0$ and, for any $\alpha>0, h_{\tau}(\alpha, 0) \rightarrow \alpha_{\max } / 2$ as $\tau \rightarrow+\infty$. In order to cover this case, one can use the following interpolation function

$$
h_{\tau}(\alpha, 0)=\widetilde{h}_{\tau}(\alpha)=2 \alpha_{\max }\left(\frac{1}{1+\exp (-\tau \alpha)}-\frac{1}{2}\right),
$$

which satisfies $\widetilde{h}_{\tau}(0)=0$ and, if $\alpha>0, \widetilde{h}_{\tau}(\alpha) \rightarrow \alpha_{\max }$ as $\tau \rightarrow+\infty$. 


\subsection{Comparison with standard RAMP interpolation function}

The RAMP function has been introduced in [4] and is defined as follows:

$$
h_{q, \mathrm{R}}(s)=\alpha_{\max }+\left(\alpha_{\min }-\alpha_{\max }\right)(1-s) \frac{1+q}{1-s+q},
$$

where $s \in[0,1]$ is the design parameter and $\alpha_{\min }, \alpha_{\max }$ are respectively the minimal and maximal value the RAMP function can take. Again, some direct computations show that $h_{q, \mathrm{R}}$ is a smooth and convex regularization of a Heaviside step function since $h_{q, \mathrm{R}}(0)=\alpha_{\text {min }}$ and, for any $s>0$, $\lim _{q \rightarrow 0} h_{q, \mathrm{R}}(s)=\alpha_{\max }$. In the sequel, we use $\alpha_{\text {min }}=0$ since this does not add any difficulties nor plus to the discussion. We now compare the performances of our sigmoid (4) with the standard RAMP function (6) by focusing on the size of their so-called transition zones. First, note that they are both defined on $\Omega$ but the transition zone only depends on the value of the design parameter $s(x)$ for $x \in \Omega$. As a result, we are going to consider that both functions have real domain and act on the interval $[0,1]$. Also, to compare functions having the same domain and being both smooth regularization of step function as $q \rightarrow 0$, we rescale the sigmoids (4) thanks to the formula

$$
h_{q, \mathrm{~S}}\left(s, \alpha_{0}\right)=h_{1 / q}\left(\alpha_{\max } s, \alpha_{0}\right), h_{q, \mathrm{~S}, 0}(s)=\widetilde{h}_{1 / q}\left(s \alpha_{\max }\right), s \in[0,1]
$$

By definition, the transition zones are the undesirable values of the design variable $s \in[0,1]$ for which the velocity of the speed is not enough penalized to vanish or the value of the interpolation function is not small enough for considering these zones as fluid. As one can see from Figure 1, the sigmoid function has much smaller transition zone than the RAMP function.

To prove this claim and get more qualitative results we are going to compute explicitly the size of these transition zones. For a given small enough 

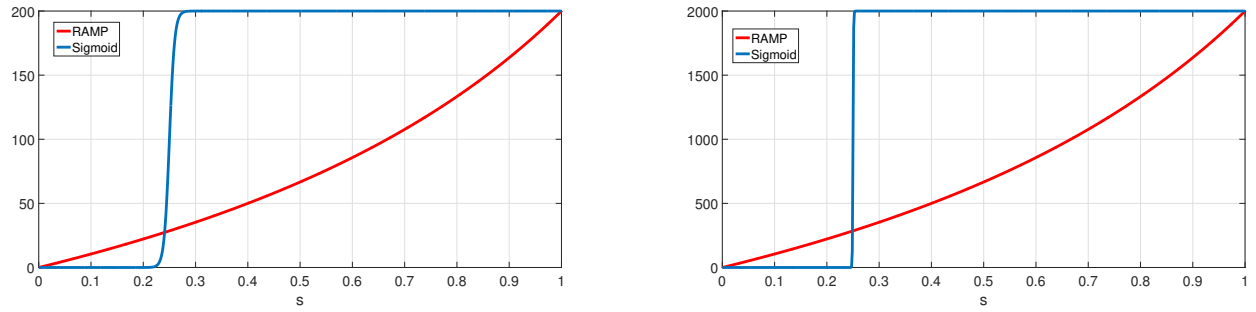

Figure 1: Interpolation functions with $q=1$ and $\alpha_{0}=\alpha_{\max } / 4$. Left: $\alpha_{\max }=200$. Right: $\alpha_{\max }=2000$.

203

204

$\varepsilon>0$ and a large enough $M>0$, the latter are defined by

$$
\mathcal{T}(f, \varepsilon, M)=\{s \in[0,1] \mid \varepsilon \leq f(s) \leq M\}, f \in\left\{h_{q, \mathrm{R}}, h_{q, \mathrm{~S}}, h_{q, \mathrm{~S}, 0}\right\}
$$

Since both interpolation function are increasing, they admit an inverse function $f^{-1}$ and the transition zone is thus given by the interval $\mathcal{T}(f, \varepsilon, M)=$ $\left[f^{-1}(\varepsilon), f^{-1}(M)\right]$ from which we infer that

$$
|\mathcal{T}(f, \varepsilon, M)|=f^{-1}(M)-f^{-1}(\varepsilon) .
$$

It then only remains to compute the inverse of the sigmoid and RAMP functions. This is actually achieved by solving $f(s)=y$ for a given $y \in\left[0, \alpha_{\max }\right]$. These equations can be solved analytically using only direct computations 
and give

$$
\begin{aligned}
& s=\left(h_{q, \mathrm{R}}\right)^{-1}(y)=1-q \frac{y-\alpha_{\max }}{\alpha_{\max }-y+(1+q)\left(\alpha_{\min }-\alpha_{\max }\right)}=y \frac{1+q}{\alpha_{\max } q+y} \\
& s=\left(h_{q, \mathrm{~S}}\right)^{-1}(y)=\frac{\alpha_{0}}{\alpha_{\max }}- \\
& \frac{q}{\alpha_{\max }}\left\{\begin{array}{l}
-\log \left(\frac{\alpha_{\max }-y\left(1+\exp \left(-\alpha_{0} / q\right)\right)}{\alpha_{\max } \exp \left(-\alpha_{0} / q\right)+y\left(1+\exp \left(-\alpha_{0} / q\right)\right)}\right) \quad \text { if } s \leq \alpha_{0} / \alpha_{\max } \\
\log \left(\frac{\alpha_{\max }-y\left(1+\exp \left(-\alpha_{0} / q\right)\right)}{\alpha_{\max } \exp \left(-\alpha_{0} / q\right)+y\left(1+\exp \left(-\alpha_{0} / q\right)\right)}\right) \quad \text { if } s \geq \alpha_{0} / \alpha_{\max }
\end{array}\right. \\
& s=\left(h_{q, \mathrm{~S}, 0}\right)^{-1}(y)=-\log \left(\frac{\alpha_{\max }-y}{\alpha_{\max }+y}\right) \frac{q}{\alpha_{\max }} .
\end{aligned}
$$

The constraint on $s$ in the definition of the inverse of $h_{q, \mathrm{~S}}$ comes from the fact that we have to solve an equation of the form $\exp \left(\left(\alpha_{0}-s \alpha_{\max }\right) / q\right)=g(y)$ for a positive function $g$. Using (7) and (8), the size of the transition zones are finally

$$
\begin{aligned}
& \left|\mathcal{T}\left(h_{q, \mathrm{R}}, \varepsilon, M\right)\right|:=\mathcal{T}_{\mathrm{R}}=q \alpha_{\max } \frac{(1+q)}{\left(\alpha_{\max } q+M\right)\left(\alpha_{\max } q+\varepsilon\right)}(M-\varepsilon), \\
& \left|\mathcal{T}\left(h_{q, \mathrm{~S}}, \varepsilon, M\right)\right|:=\mathcal{T}_{\mathrm{S}}=\frac{q}{\alpha_{\max }} \log \left(\frac{\alpha_{\max }-M\left(1+\exp \left(-\alpha_{0} / q\right)\right)}{\alpha_{\max } \exp \left(-\alpha_{0} / q\right)+M\left(1+\exp \left(-\alpha_{0} / q\right)\right)}\right) \\
& +\frac{q}{\alpha_{\max }} \log \left(\frac{\alpha_{\max }-\varepsilon\left(1+\exp \left(-\alpha_{0} / q\right)\right)}{\alpha_{\max } \exp \left(-\alpha_{0} / q\right)+\varepsilon\left(1+\exp \left(-\alpha_{0} / q\right)\right)}\right) \\
& \left|\mathcal{T}\left(h_{q, \mathrm{~S}, 0}, \varepsilon, M\right)\right|:=\mathcal{T}_{\mathrm{S}, 0}=\frac{q}{\alpha_{\max }} \log \left(\frac{\left(\alpha_{\max }-M\right)\left(\alpha_{\max }+\varepsilon\right)}{\left(\alpha_{\max }+M\right)\left(\alpha_{\max }-\varepsilon\right)}\right) .
\end{aligned}
$$

For $\alpha_{0}=0$, one can clearly see that $\mathcal{T}_{\mathrm{S}, 0}<\mathcal{T}_{\mathrm{R}}$. To deal with the case $\alpha_{0}>0$, note that the parameter $q$ is small since we wish the interpolation function to be close to an ideal step function. Also, we emphasize that $\alpha_{0}>0$ is going to depend on $\alpha_{\max }$ in order to control the percentage of fluid in $\Omega$. Since $\alpha_{\max }$ has to be large enough, one gets that $z=\exp \left(-\alpha_{0} / q\right)$ is a small parameter 
of the RAMP function. Some numerical calculation of the size of transition

\begin{tabular}{|c|c|c|c|}
\hline & $\mathcal{T}_{\mathrm{R}}$ & $\mathcal{T}_{\mathrm{S}}$ & $\mathcal{T}_{\mathrm{S}, 0}$ \\
\hline$q=1$ & 0.6657 & 0.0380 & 0.0055 \\
\hline$q=10^{-4}$ & 0.1665 & $3.8002 \times 10^{-6}$ & $5.4881 \times 10^{-7}$ \\
\hline$q=10^{-5}$ & 0.0196 & $3.8002 \times 10^{-7}$ & $5.4881 \times 10^{-8}$ \\
\hline
\end{tabular}

Table 1: Size of the transition zones for $\alpha_{\max }=200, M=\alpha_{\max } / 2, \varepsilon=0.1$ and $\alpha_{0}=$ $\alpha_{\max } / 4$.

\begin{tabular}{|c|c|c|c|}
\hline & $\mathcal{T}_{\mathrm{R}}$ & $\mathcal{T}_{\mathrm{S}}$ & $\mathcal{T}_{\mathrm{S}, 0}$ \\
\hline$q=1$ & 0.6666 & 0.0050 & $5.4926 \times 10^{-4}$ \\
\hline$q=10^{-4}$ & 0.6665 & $4.9517 \times 10^{-7}$ & $5.4926 \times 10^{-8}$ \\
\hline$q=10^{-5}$ & 0.1666 & $4.9517 \times 10^{-8}$ & $5.4926 \times 10^{-9}$ \\
\hline
\end{tabular}

Table 2: Size of the transition zones for $\alpha_{\max }=2000, M=\alpha_{\max } / 2, \varepsilon=0.1$ and $\alpha_{0}=$ $\alpha_{\max } / 4$.

235

$$
\mathcal{T}_{\mathrm{S}}=\frac{q}{\alpha_{\max }} \log \left(\frac{\left(\alpha_{\max }-M\right)\left(\alpha_{\max }-\varepsilon\right)}{M \varepsilon}\right)+O\left(\exp \left(-\alpha_{0} / q\right)\right),
$$

from which one can see that $\mathcal{T}_{\mathrm{S}}<\mathcal{T}_{\mathrm{R}}$. Thanks to the factor $q / \alpha_{\max }$, we can see that the intermediate zones of the sigmoid are in fact much smaller than those $\alpha_{\max } / 4$.

zone confirming this fact can be found in Table 1,2 for $\alpha_{\max } \in\{200,2000\}$ and $q \in\left\{1,10^{-4}, 10^{-5}\right\}$.

and we can thus expand $\mathcal{T}_{\mathrm{S}}$ as $z \rightarrow 0$. This gives 


\subsection{Interpolation of the thermal conductivity}

The effective conductivity is interpolated using a sigmoid function similar to $(4)$ :

$$
k_{\tau}\left(\alpha, \alpha_{0}\right)=\left(k_{s}-k_{f}\right)\left(\frac{1}{1+\exp \left(-\tau\left(\alpha-\alpha_{0}\right)\right)}-\frac{1}{1+\exp \left(\tau \alpha_{0}\right)}\right)+k_{f}
$$

where $\alpha \in\left[0, \alpha_{\max }\right]$ is the design parameter, $k_{s}$ and $k_{f}$ are respectively the adimensional thermal diffusivity of the solid and of the fluid. Interpolation function (9) is again a smooth regularization of a Heaviside step function that satisfies $k_{\tau}\left(0, \alpha_{0}\right)=k_{f}$ together with the point-wise convergence

$$
\lim _{\tau \rightarrow+\infty} k_{\tau}\left(\alpha, \alpha_{0}\right)= \begin{cases}k_{f} & \text { if } \alpha<\alpha_{0}, \\ \left(k_{f}+k_{s}\right) / 2 & \text { if } \alpha=\alpha_{0}, \\ k_{s} & \text { if } \alpha>\alpha_{0} .\end{cases}
$$

Similarly to $h_{\tau}$, the case $\alpha_{0}=0$ needs the following slight modification to still have, when $\tau \rightarrow+\infty$, a fluid-solid step function

$$
k_{\tau}(\alpha, 0)=\widetilde{k}_{\tau}(\alpha)=2\left(k_{s}-k_{f}\right)\left(\frac{1}{1+\exp (-\tau \alpha)}-\frac{1}{2}\right)+k_{f} .
$$

The previous function then satisfies $\widetilde{k}_{\tau}(0)=0$ and, for any $\alpha>0, \widetilde{k}_{\tau}(\alpha) \rightarrow k_{s}$ as $\tau \rightarrow+\infty$. A RAMP-like interpolation function is also usually used for the thermal conductivity $[30,15]$. Computations similar to those of Section 3.2 can be done to prove that the transition zone of the sigmoid (9) are much smaller than those of the RAMP function hence motivating using such technique in topology optimization. 


\section{Gradient computation with the adjoint model}

We chose to solve the optimization problem (1) with gradient-based optimization algorithm. The latter requires the computation of the gradient of the cost functional with respect to the design variable which needs the sensitivities of the solution to the state equation with respect to $\alpha$. To do this, we are going to use the adjoint method (see e.g. $[24,12,45]$ ) since it allows to compute the gradient of $\mathcal{J}$ efficiently, only by solving the so-called direct problem (the governing equation) and the adjoint model.

We adopt here the differentiate then discretize approach [25] which means that we need to compute the continuous adjoint model associated to (1) as done in $[17,18]$. The Lagrangian associated to the optimization problem (1) can then be defined as follows

$$
\begin{aligned}
& \mathcal{L}\left((\mathbf{u}, \theta, p),\left(\mathbf{u}^{*}, \theta^{*}, p^{*}\right), \alpha\right):=\mathcal{J}(\mathbf{u}, \theta, p)-\int_{\Omega} p^{*} \operatorname{div} \mathbf{u} d \Omega \\
& -\int_{\Omega} \mathbf{u}^{*} \cdot\left[(\mathbf{u} \cdot \nabla) \mathbf{u}+\nabla p-\operatorname{Re}^{-1} \Delta \mathbf{u}+h_{\tau}\left(\alpha, \alpha_{0}\right) \mathbf{u}-\operatorname{Ri} \theta \overrightarrow{e_{y}}\right] d \Omega \\
& -\int_{\Omega} \theta^{*} \cdot\left[\nabla \cdot(\mathbf{u} \theta)-\operatorname{div}\left(\operatorname{Re}^{-1} \operatorname{Pr}^{-1} k_{\tau}\left(\alpha, \alpha_{0}\right) \nabla \theta\right)\right] d \Omega \\
& -\int_{\Gamma_{1}}\left(\mathbf{u} \cdot \Phi_{1}+\left(\partial_{n} \theta-1\right) \psi_{1}\right) d \Gamma-\int_{\Gamma_{2}}\left(\mathbf{u} \cdot \Phi_{2}+\partial_{n} \theta \psi_{2}\right) d \Gamma \\
& -\int_{\Gamma_{\text {in }}}\left(\left(\mathbf{u}-\mathbf{u}_{\text {in }}\right) \cdot \Phi_{\text {in }}+\theta \psi_{\text {in }}\right) d \Gamma-\int_{\Gamma_{\text {out }}}\left(\partial_{n} \mathbf{u} \cdot \Phi_{\text {out }}+p q_{\text {out }}+\partial_{n} \theta \psi_{\text {out }}\right) d \Gamma
\end{aligned}
$$

where $\left(\mathbf{u}^{*}, \theta^{*}, p^{*}, \Phi_{l}, \psi_{l}, q_{\text {out }}\right)$, for $l \in\{1,2$, in, out $\}$, can be seen as Lagrange multiplier (or adjoint variables). We emphasize that the variables $\left(\Phi_{t}, \psi_{t}, q_{\text {out }}\right)$ are here to enforce the boundary conditions (3).

The adjoint model is defined thanks to the critical point of $\mathcal{L}$ with respect to the state variables $(\mathbf{u}, \theta, p)$. We use below the following notation for the derivative of an application $\mathcal{F}: x \in \mathbf{E} \mapsto \mathcal{F}(x) \in \mathbf{F}$, where $\mathbf{E}, \mathbf{F}$ are normed 
spaces

$$
\frac{\partial \mathcal{F}}{\partial x}[\delta x]:=\lim _{\varepsilon \rightarrow 0} \frac{\mathcal{F}(x+\varepsilon \delta x)-\mathcal{F}(x)}{\varepsilon} .
$$

First, since $\mathcal{L}$ is linear with respect to the adjoint variables, it is worth noting that we recover the state equation (2)-(3) if, for $l \in\{1,2$, in, out $\}$, we solve

$$
\frac{\partial \mathcal{L}}{\partial\left(\mathbf{u}^{*}, \theta^{*}, p^{*}, \Phi_{l}, \psi_{l}, q_{\text {out }}\right)}\left[\delta \mathbf{u}^{*}, \delta \theta^{*}, \delta p^{*}, \delta \Phi_{l}, \delta \psi_{l}, \delta q_{\text {out }}\right]=0 .
$$

Using now (11) to differentiate the Lagrangian (10) with respect to the state variables, and integrating by parts to have no terms involving derivative of $(\delta \mathbf{u}, \delta \theta, \delta p)$, we end up with

$$
\begin{aligned}
& \frac{\partial \mathcal{L}}{\partial(\mathbf{u}, \theta, p)}[\delta \mathbf{u}, \delta \theta, \delta p]=\int_{\Omega} \frac{\partial \mathcal{J}_{\Omega}}{\partial(\mathbf{u}, \theta, p)}[\delta \mathbf{u}, \delta \theta, \delta p] d \Omega+\int_{\Gamma} \frac{\partial \mathcal{J}_{\Gamma}}{\partial(\mathbf{u}, \theta, p)}[\delta \mathbf{u}, \delta \theta, \delta p] d \Gamma+ \\
& \int_{\Omega} \delta \mathbf{u} \cdot\left(\nabla p^{*}-h_{\tau}\left(\alpha, \alpha_{0}\right) \mathbf{u}^{*}-(\nabla \mathbf{u})^{T} \mathbf{u}^{*}+\nabla \mathbf{u}^{*} \mathbf{u}+\theta \nabla \theta^{*}+R e^{-1} \Delta \mathbf{u}^{*}\right) d \Omega \\
& +\int_{\Omega} \delta \theta\left(\operatorname{div}\left(\operatorname{Re}^{-1} \operatorname{Pr}^{-1} k_{\tau}\left(\alpha, \alpha_{0}\right) \nabla \theta^{*}\right)+\mathbf{u} \cdot \nabla \theta^{*}+R i \mathbf{u}^{*} \cdot \mathbf{e}_{\mathbf{y}}\right) d \Omega \\
& +\int_{\Omega} \delta p \operatorname{div} \mathbf{u}^{*} d \Omega-\int_{\Gamma} \delta p\left(\mathbf{u}^{*} \cdot n\right) d \Gamma \\
& +\int_{\Gamma} \delta \mathbf{u} \cdot\left(-n p^{*}-n \theta \theta^{*}+\operatorname{Re}^{-1} \partial_{n} \mathbf{u}^{*}-(\mathbf{u} \cdot n) \mathbf{u}^{*}\right) d \Gamma-\int_{\Gamma} R^{-1} \partial_{n} \delta \mathbf{u} \cdot \mathbf{u}^{*} d \Gamma \\
& +\int_{\Gamma} \delta \theta\left((-\mathbf{u} \cdot n) \theta^{*}-\operatorname{Re}^{-1} \operatorname{Pr}^{-1} k_{\tau}\left(\alpha, \alpha_{0}\right) \partial_{n} \theta^{*}\right) d \Gamma+\int_{\Gamma} \theta^{*} \operatorname{Re}^{-1} \operatorname{Pr}^{-1} k_{\tau}\left(\alpha, \alpha_{0}\right) \partial_{n} \delta \theta d \Gamma \\
& -\int_{\Gamma_{1}}\left(\delta \mathbf{u} \cdot \Phi_{1}+\partial_{n} \delta \theta \psi_{1}\right) d \Gamma-\int_{\Gamma_{2}}\left(\delta \mathbf{u} \cdot \Phi_{2}+\partial_{n} \delta \theta \psi_{2}\right) d \Gamma \\
& -\int_{\Gamma_{\text {in }}}\left(\delta \mathbf{u} \cdot \Phi_{\text {in }}+\delta \theta \psi_{\text {in }}\right) d \Gamma-\int_{\Gamma_{\text {out }}}\left(\partial_{n} \delta \mathbf{u} \cdot \Phi_{\text {out }}+\delta p q_{\text {out }}+\partial_{n} \delta \theta \psi_{\text {out }}\right) d \Gamma
\end{aligned}
$$

Assuming that $\left(\delta \mathbf{u}, \delta \theta, \delta p, \Phi_{l}, \psi_{l}, q_{\text {out }}\right)=0$ for $l \in\{1,2$, in, out $\}$, that the first derivatives of $\delta \mathbf{u}, \delta \theta$ vanish on $\Gamma$ and solving $(\partial \mathcal{L} / \partial(\mathbf{u}, \theta, p))[\delta \mathbf{u}, \delta \theta, \delta p]=0$ 
yield the adjoint problem:

$$
\begin{aligned}
& \nabla p^{*}-h_{\tau}(\alpha) \mathbf{u}^{*}+\theta \nabla \theta^{*}+R e^{-1} \Delta \mathbf{u}^{*}+\nabla \mathbf{u}^{*} \mathbf{u}-(\nabla \mathbf{u})^{T} \mathbf{u}^{*}=-\frac{\partial \mathcal{J}_{\Omega}}{\partial \mathbf{u}} \quad \text { in } \Omega \\
& \operatorname{div} \mathbf{u}^{*}=-\frac{\partial \mathcal{J}_{\Omega}}{\partial p} \quad \text { in } \Omega \\
& R i \mathbf{u}^{*} \cdot \overrightarrow{e_{y}}+\mathbf{u} \cdot \nabla \theta^{*}+\operatorname{div}\left(\operatorname{Re}^{-1} \operatorname{Pr}^{-1} k_{\tau}(\alpha) \nabla \theta^{*}\right)=-\frac{\partial \mathcal{J}_{\Omega}}{\partial \theta} \quad \text { in } \Omega \text {. }
\end{aligned}
$$

For the adjoint boundary condition, we are going to show how to obtain them on $\Gamma_{1} \cup \Gamma_{2}$ since the other part of $\Gamma$ can be done in the same spirit. On both walls, the primal velocity is fixed to zero (no-slip condition on the walls) and the heat flux either vanishes or is constant. Since div $\mathbf{u}=0$ and $\mathbf{u}$ is prescribed or null value, there will be $\delta \mathbf{u}=0$, div $\delta \mathbf{u}=0$ and $\partial_{n} \delta \theta=0$. We recall a formula from [11, Lemma 7] that holds for any vector fields w and reads

$$
\partial_{n} \mathbf{w} \cdot n=\left.\operatorname{div} \mathbf{w}\right|_{\Gamma}-\operatorname{div}_{\Gamma} \mathbf{w}_{t}-\kappa \mathbf{w} \cdot n
$$

where $\kappa=\operatorname{div} n$ is the curvature of $\Gamma$ and $\operatorname{div}_{\Gamma}$ is the surface divergence operator. Since $\partial_{n} \delta \mathbf{u} \cdot \mathbf{u}^{*}=\left(\partial_{n} \delta \mathbf{u}\right)_{n} \cdot u_{n}^{*}+\left(\partial_{n} \delta \mathbf{u}\right)_{t} \cdot u_{t}^{*}$ and $\delta \mathbf{u}=0$ and $\operatorname{div} \delta \mathbf{u}=0$, formula (14) gives that

$$
\partial_{n} \delta \mathbf{u} \cdot \mathbf{u}^{*}=\left(\partial_{n} \delta \mathbf{u}\right)_{t} u_{t}^{*}
$$

The boundary conditions on $\Gamma_{1} \cup \Gamma_{2}$ are then obtained from (12) by considering, for $l=1,2$, the following vanishing terms

$$
\begin{aligned}
& \int_{\Gamma_{l}} R e^{-1} \partial_{n} \delta \mathbf{u} \cdot \mathbf{u}^{*} d \Gamma=\int_{\Gamma} \operatorname{Re}^{-1}\left(\partial_{n} \delta \mathbf{u}\right)_{t} \cdot u_{t}^{*} d \Gamma=0 \\
& \int_{\Gamma_{l}} \delta p\left(\mathbf{u}^{*} \cdot n-\frac{\partial \mathcal{J}_{\Gamma}}{\partial p}\right) d \Gamma=0 \\
& \int_{\Gamma_{l}} \delta \theta\left((-\mathbf{u} \cdot n) \theta^{*}-\operatorname{Re}^{-1} \operatorname{Pr}^{-1} k_{\tau}\left(\alpha, \alpha_{0}\right) \partial_{n} \theta^{*}+\frac{\partial \mathcal{J}_{\Gamma}}{\partial \theta}\right) d \Gamma=0 .
\end{aligned}
$$


The adjoint boundary conditions on $\Gamma_{i n}$ are obtained as above by taking $\delta \theta=0$. For the outlet, we use $\partial_{n} \delta \theta=0, \partial_{n} \delta \mathbf{u}=0$ and $\delta p=0$. Finally, we end up with the adjoint boundary condition

$$
\begin{array}{ll}
\text { On } \Gamma_{1} \cup \Gamma_{2}: & u_{t}^{*}=0, u_{n} \theta^{*}+\operatorname{Re}^{-1} \operatorname{Pr}^{-1} k_{\tau}\left(\alpha, \alpha_{0}\right) \partial_{n} \theta^{*}=\frac{\partial \mathcal{J}_{\Omega}}{\partial \theta}, u_{n}^{*}=\frac{\partial \mathcal{J}_{\Omega}}{\partial p}, \\
\text { On } \Gamma_{\text {in }}: & u_{n}^{*}=\frac{\partial \mathcal{J}_{\Gamma}}{\partial p} u_{t}^{*}=0, \theta^{*}=0, \\
\text { On } \Gamma_{\text {out }}: & u_{n} \theta^{*}+\operatorname{Re}^{-1} \operatorname{Pr}^{-1} k_{\tau}\left(\alpha, \alpha_{0}\right) \partial_{n} \theta^{*}=\frac{\partial \mathcal{J}_{\Gamma}}{\partial \theta}, \\
& n p^{*}+n \theta \theta^{*}+\operatorname{Re}^{-1} \partial_{n} u^{*}+u_{n} \mathbf{u}^{*}=\frac{\partial \mathcal{J}_{\Gamma}}{\partial \mathbf{u}} .
\end{array}
$$

$$
\text { Now solving }(\partial \mathcal{L} / \partial \alpha)[\delta \alpha]=0 \text { for all } \delta \alpha \text { yields }
$$

$$
\begin{aligned}
& \frac{\partial h_{\tau, \alpha_{0}}}{\partial \alpha} \mathbf{u} \cdot \mathbf{u}^{*}+\operatorname{Re}^{-1} \operatorname{Pr}^{-1} \frac{\partial k_{\tau, \alpha_{0}}}{\partial \alpha} \nabla \theta \cdot \nabla \theta^{*}=0 \quad \text { in } \Omega \\
& \frac{\partial k_{\tau, \alpha_{0}}}{\partial \alpha} \theta^{*}=0 \quad \text { on } \Gamma_{1},
\end{aligned}
$$

which is the optimality condition. Finally, according to the adjoint method (see e.g. $[24,12,25])$, the gradient of the cost functional $\mathcal{J}(\mathbf{u}, \theta, p)$ at some $\alpha$ is given by

312

$$
\begin{array}{ll}
\frac{\partial \mathcal{J}}{\partial \alpha}(\alpha)=\frac{\partial h_{\tau, \alpha_{0}}}{\partial \alpha} \mathbf{u} \cdot \mathbf{u}^{*}+\operatorname{Re}^{-1} \operatorname{Pr}^{-1} \frac{\partial k_{\tau, \alpha_{0}}}{\partial \alpha} \nabla \theta \cdot \nabla \theta^{*}, & \text { in } \Omega, \\
\frac{\partial \mathcal{J}}{\partial \alpha}(\alpha)=\frac{\partial k_{\tau, \alpha_{0}}}{\partial \alpha} \theta^{*} & \text { on } \Gamma_{1},
\end{array}
$$

where $(\mathbf{u}, \theta, p)$ satisfy $(2-3)$ and $\left(\mathbf{u}^{*}, \theta^{*}, p^{*}\right)$ satisfy the adjoint problem (13$16)$.

Remark 1. The adjoint variables $\left(\Phi_{l}, \psi_{l}, p_{\text {out }}\right)$ used to enforce the boundary conditions (3) of the primal problem are not needed to solve the adjoint problem. Nevertheless, they can be determined using $(13,15)$ which cancel many 
terms in (12) and gives

$$
\begin{aligned}
& \Phi_{l}=-n p^{*}-n \theta \theta^{*}+R e^{-1} \partial_{n} \mathbf{u}^{*}-(\mathbf{u} \cdot n) \mathbf{u}^{*}+\frac{\partial \mathcal{J}_{\Gamma}}{\partial \mathbf{u}} \text { on } \Gamma_{l} \\
& \psi_{l}=\theta^{*} \operatorname{Re}^{-1} \operatorname{Pr}^{-1} k_{\tau}\left(\alpha, \alpha_{0}\right)+\frac{\partial \mathcal{J}_{\Gamma}}{\partial \theta} \text { on } \Gamma_{l}, l \in\{1,2, \text { in }\} \\
& \Phi_{\text {out }}=-\operatorname{Re}^{-1} \mathbf{u}^{*}, \psi_{\text {out }}=\theta^{*} \operatorname{Re}^{-1} \operatorname{Pr}^{-1} k_{\tau}\left(\alpha, \alpha_{0}\right), q_{\text {out }}=-u_{n}^{*}-\frac{\partial \mathcal{J}_{\Gamma}}{\partial p} .
\end{aligned}
$$

Note finally that these adjoint variables are also not needed to compute the gradient of the cost functional with respect to the design variable since they does not appear in (18).

\section{Numerical example}

This section aims to validate the new interpolation technique. To do so, we investigate two objective functions. First objective function is related to the power dissipated by the fluid through the domain $\Omega$ and can be evaluated on the basis of total pressure losses as follows:

$$
\mathcal{J}_{1}(\mathbf{u}, p, \theta)=-\int_{\Gamma} p_{t} \mathbf{u} \cdot \mathbf{n} d \Gamma \quad \text { where } p_{t}=p+\frac{1}{2} \mathbf{u}^{2}
$$

The second cost function is related to the maximization of the recoverable thermal power from the domain $\Omega$ and is given by

$$
\mathcal{J}_{2}(\mathbf{u}, p, \theta)=\int_{\Gamma} \theta \mathbf{u} \cdot \mathbf{n} d \Gamma
$$

We finally consider the following objective functional

$$
\begin{aligned}
\operatorname{minimize:~} & \\
\mathcal{J}(\mathbf{u}, p, \theta) & =\mathcal{J}_{1}(\mathbf{u}, p, \theta)-\mathcal{J}_{2}(\mathbf{u}, p, \theta), \\
& =-\int_{\Gamma}\left(p_{t}+\theta\right) \mathbf{u} \cdot \mathbf{n} d \Gamma
\end{aligned}
$$

subject to: Governing equation (2),

Boundary conditions (3). 

mization is described in Table 3. The forward problem and the optimization

Step 0. Initialization: set all the constants $R e, R i, \operatorname{Pr}$

Step 1. Solve the forward problem (2),(3) problem with the Finite Volume Method

Step 2. Compute objective and constraint values

Step 3. Compute sensitivities by adjoint method

Step 4. Evaluate the optimality condition. If a stopping criterion is met, terminate the calculation Step 5. Update design variables $\alpha$ with $\alpha_{k+1}=-\nabla \mathcal{J}_{k+1}+\beta_{k+1}^{P R} \alpha_{k}$ and return to step 1

Table 3: Algorithm of topology optimization

processes are implemented using OpenFOAM [44]. In Step 5, the design 
variables are evaluated by using the conjugated-gradient descent direction method associated to Polack-Ribiere method $\beta_{k+1}^{P R}=\frac{\nabla \mathcal{J}_{k+1}^{T}\left(\nabla \mathcal{J}_{k+1}-\nabla \mathcal{J}_{k}\right)}{\nabla \mathcal{J}_{k}^{T} \nabla \mathcal{J}_{k}}$.

After optimization process, several results are compared as optimized designs, velocity magnitude distribution, temperature distribution, and lastly, we focus on fluid-solid interfaces. We also presents the objective function values for each simulation case. Finally, we investigate to vary the slope abscissa and the curve of sigmoid functions in order to evaluate their impact on the optimization problem. That corresponds to consider different values of $\alpha_{0}$ and $\tau$. In our study, $\alpha_{0}$ will vary in $\{25,50,100\}$ which correspond respectively to $\left\{\alpha_{\max } / 8, \alpha_{\max } / 4, \alpha_{\max } / 2\right\}$ and $\tau$ will vary in $\{0.3,0.7,1.0\}$. In order to perform topology optimization, the $\alpha$ term may take a finite value between 0 and $\alpha_{\max }=200$.

\subsection{Studied Cases}

Two representative cases of topology optimization in fluid dynamics are investigated : the single pipe case and the bend pipe case. Figure 2 shows schematic illustrations of the two studied cases.

\subsubsection{Design of a single pipe.}

We present here a benchmark numerical example used recently by Marck [15] to illustrate the viability and efficiency of the methodology presented in this paper. The design domain consists in a cavity of side $L$. The computational domain is discretized using $100 \times 100$ elements. The inlet flow is located in the center of the west edge for a length of $L / 5$. The outlet flow is lining up with it on the east edge. The rest of west and east edges are assumed to be adiabatic and will be called $\Gamma_{2}$, whereas the whole north and 

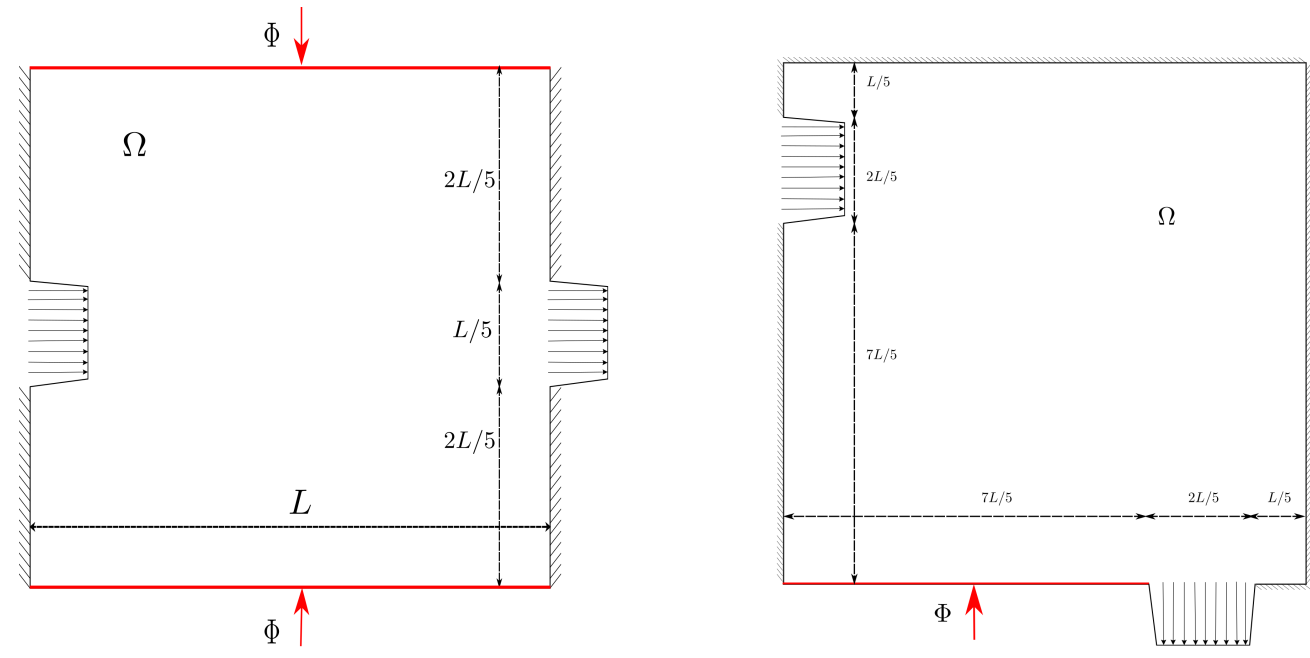

Figure 2: Single pipe and Bend pipe

374

south walls are subjected to a constant flux of temperature, called thereafter $\Gamma_{1}$.

\subsubsection{Design of a bend pipe.}

The second example has been studied by several authors as [4] and [15]. The computational domain is also square-shaped, with a adimensional side $L=1$, and the design grid is made of $100 \times 100$ elements. The inlet flow is located at $L / 5$ of the west edge. The outlet flow is located on the south edge, at $L / 5$ from the east edge. The length of both flow boundary conditions is set to $2 L / 5$. The part of the south edge located on the left of the outlet is subjected to a constant flux of temperature and will be called $\Gamma_{1}$. The rest of the edges are assumed to be adiabatic and will be designated as $\Gamma_{2}$.

\subsubsection{Physical parameters.}

For the two representative cases studied in this section, the inlet flow is prescribed with a constant horizontal velocity equal to $U_{0}=0.04 \mathrm{~m} . \mathrm{s}^{-1}$ 
used in Reynolds number. The inlet temperature is fixed to $\theta=0$. The outflow temperature condition is fixed to a zero gradient, as well as the velocity gradient. Hot walls are subject to a constant flux of temperature $\partial_{n} \theta=-1$. The Reynolds number based on the characteristic dimension of the inlet length and the average inlet velocity is $R e=500$. The Rayleigh number is fixed to $R a=5 \times 10^{5}$. Prandtl number is set to 0.71 . Grashof number has the following expression $G r=R a / P r$. Adimensional thermal parameters are the following: $k_{s}=\left(9.88 \times 10^{-5} \mathrm{~m}^{2} \cdot \mathrm{s}^{-1}\right) /\left(2.25 \times 10^{-5} \mathrm{~m}^{2} \cdot \mathrm{s}^{-1}\right)$ [(Aluminum/Air) Diffusivities], $k_{f}=1$.

\subsection{Results}

The aim of the problems defined above is to determine the optimal design that connects the inlet to the outlet of the cavity and that minimizes the objective function $\mathcal{J}$ subjected to the constraints (2) and (3).

First of all, we compute $\mathcal{J}$ without optimization for the two studied cases and we obtained respectively for the single pipe $J=9.0710^{-3}$ and for the bend pipe $J=6.3810^{-3}$. Compared to values issued from optimization process and referenced in Figure 8 and 11, they are reduced between $23 \%$ and $42 \%$ for the single pipe case, and between $18 \%$ and $19 \%$ for the bend pipe. So, the new method investigated here allows to both minimize the power dissipated by the fluid through the domain $\Omega$ and maximize the recoverable thermal power from the domain to the fluid.

Secondly, we plot velocity magnitude for various $\alpha_{0}$ and $\tau$ values. After optimization of $\mathcal{J}$ in the single pipe, we obtain an optimized design in which fluid is transported through the single pipe in a straight pipe (see Figure 3). It is quite closed to the optimized design obtained by [15] when the mechanical 

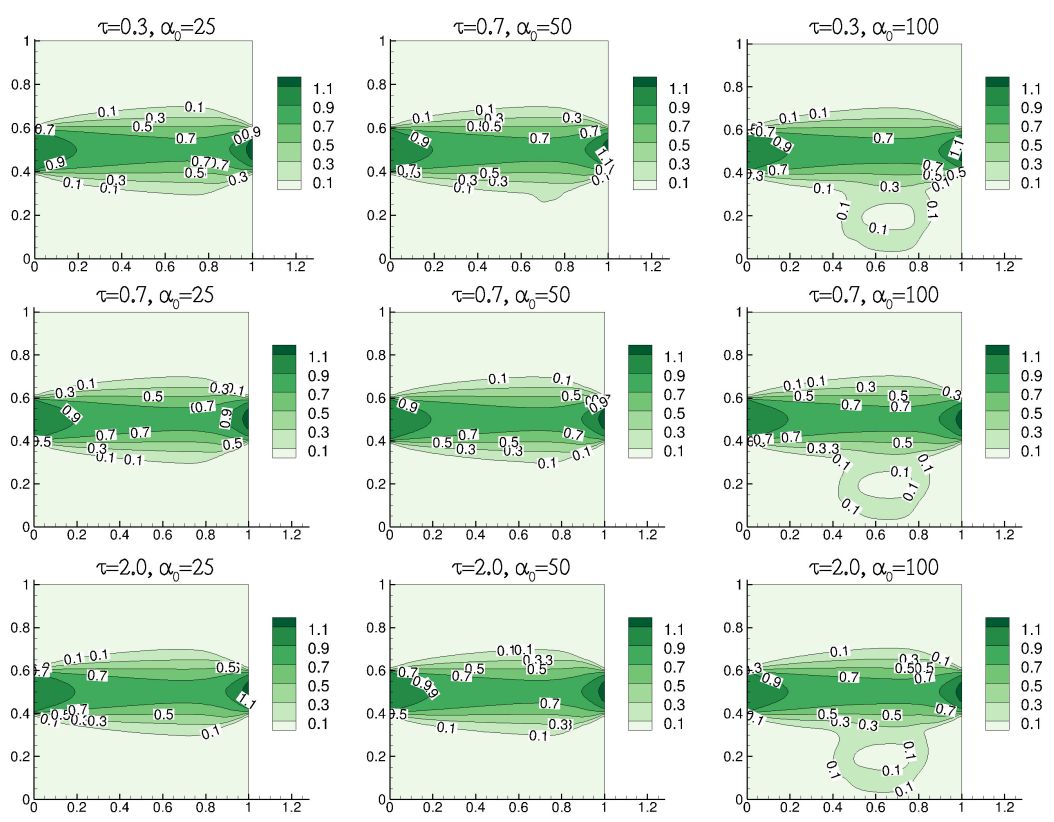

Figure 3: Velocity magnitude distribution in the single pipe

power and thermal power are considered with the same weighting coefficient. Similarly, fluid circulation forms a half circle whose center would be the left bottom corner of the square domain in the optimized bend pipe (see Figure 4). Contrary to the literature in fluid mechanics, the optimized path obtained for the flow is not as straight as possible. It can be explained by the fact that we consider here a heat transfer problem. So, the optimized pipe is larger and form a real bend because the fluid flow moves away from hot regions.

Thereafter, we compared distribution of the parameter $\alpha$ in the domain for the two studied cases (see Figures 6 and 9). We recall that values less than $\alpha_{0}$ are considered as fluid regions. We can notice that this method based on $\alpha_{0}$ affected some values of $\alpha$ to the fluid regions, which would not be possible with other methods of the literature. Indeed, generally with the 

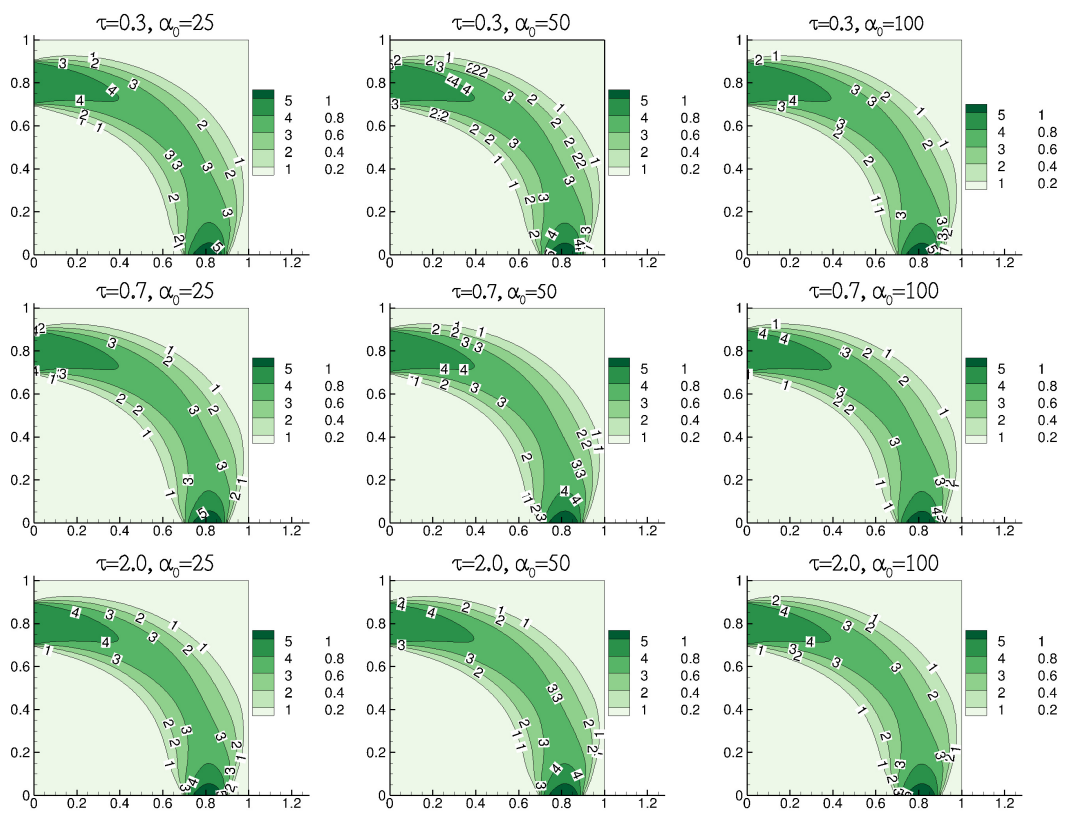

Figure 4: Velocity magnitude distribution in the bend pipe

function (6), only value of $\alpha$ equal to zero is considered as fluid element. As can be seen on these figures, intermediate values for the fluid region exist. For example, we have different values of $\alpha$ between 0 and $\alpha_{0}$ at right-top corner in Figure 9, or below the straight pipe for $\alpha_{0}=25$ in Figure 6 .

As expected, the interpolation function $h_{\tau}(\alpha)$ suppresses these intermediate values noticed previously and affects these volume elements to pure fluid volume elements. Indeed, Figures 7 and 10 show the optimized designs for various $\alpha_{0}$ and $\tau$ numbers. Compared to $\alpha$ distribution (Figures 6 and 9), they demonstrate that the interpolation function $h_{\tau}(\alpha)$ suppresses intermediate values corresponding neither to pure fluid nor to pure solid regions by affecting them to solid regions or fluid regions. However, in the bend pipe, a porous domain still exists near hot regions. Although fluid does not circulate 

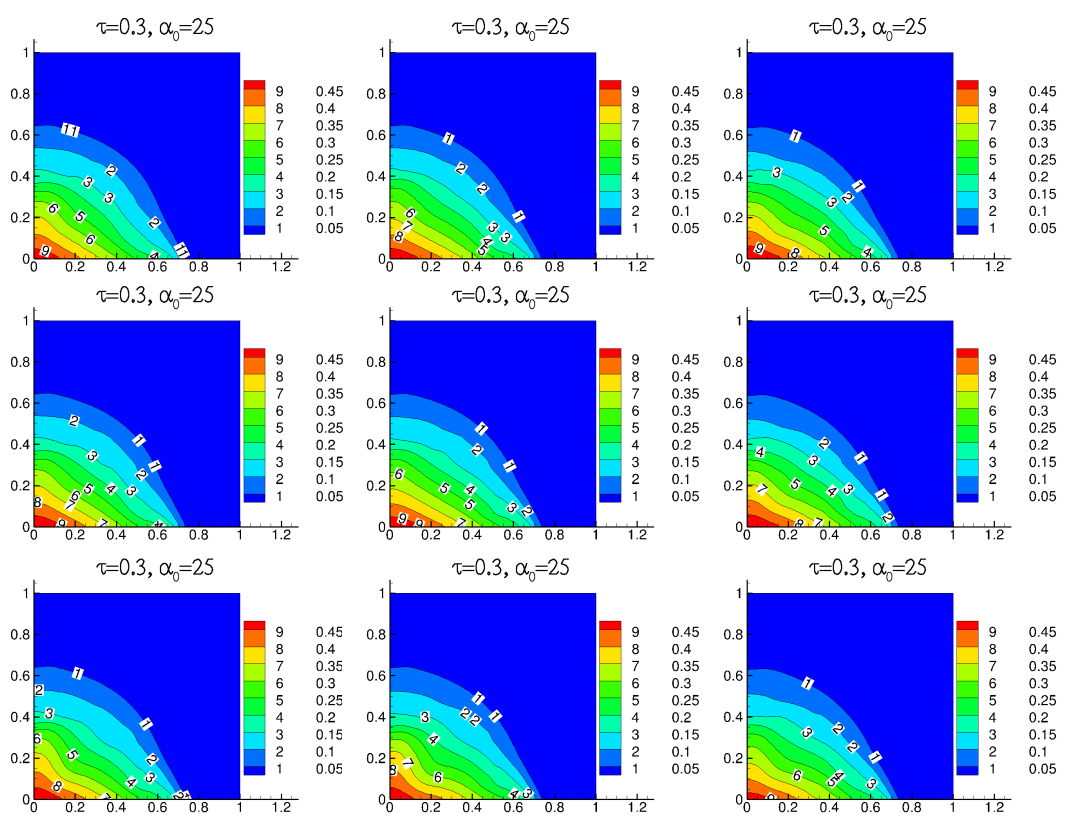

Figure 5: Temperature distribution in the bend pipe

in this porous domain, its existence contributes to increasing thermal power recovered by the fluid. That is illustrated in Figure 5 .

At last, it can be seen that thermal conductivity is well distributed, continuously, between fluid and solid regions. Figure 8 represents the distribution of material conductivity after the optimization process in the single pipe case. Compared to Figure 7, we observe that frontier between the two regions, fluid and solid, is well-established. For the bend pipe case, although intermediate regions exist, $k_{\tau}$ interpolation function allows to affect to this porous domain a conductivity closed to the fluid diffusivity (see Figure 11).

Therefore, the function $k_{\tau}(\alpha)$ provides a continuous transition between the solid and the fluid domains. 


\subsubsection{Influence of $\alpha_{0}$.}

It can clearly be seen from Figures 7 and 10 that different designs are obtained for the different values of $\alpha_{0}$. As detailed in section $3, \alpha_{0}$ is depending of $\alpha_{\max }$ in order to control the percentage of fluid in $\Omega$. This parameter has a significant impact on the quantity of material adding in the domain and that can be illustrated by computing the proportion $Q_{t}$ of material added in the domain $\Omega$ as follows :

$$
Q_{t}=\frac{\int_{\Omega} h_{\tau}(\alpha) d \Omega}{\alpha_{\max } V_{\text {tot }}} \text { where } V_{\text {tot }} \text { is the total volume of } \Omega
$$

Calculation results are referenced in Figures 7 and 10. In the single pipe, when $\alpha_{0}$ increases, quantity of material diminishes between $27.5 \%$ and $28 \%$. That means fluid domain is more important and that contributes to the increase of $\mathcal{J}_{2}$. So, that will influence the value of $\mathcal{J}$. In the bend pipe, when $\alpha_{0}$ increases, quantity of material also diminishes between $3.6 \%$ and $16 \%$. So, fluid domain is relatively more important than solid domain but not enough to significantly impact on the value of $\mathcal{J}$, hence, the small effect on values of $\mathcal{J}$ (see Figure 11). So, parameter $\alpha_{0}$ has a significant incidence on the proportion of fluid domain relatively to solid domain and therefore, can influence the value of the functional objective.

Futhermore, we observe a recirculation zone for $\alpha_{0}=100$ in the example of the optimized single pipe (see Figure 3). That can also be explained by the value of $\alpha_{0}$. Indeed, larger $\alpha_{0}$ means that the proportion of fluid elements compared to $\alpha_{\max }$ is bigger and thus fluid regions are more important. When $\alpha_{0}=100$, that means $\alpha_{0}=\alpha_{\max } / 2$, the algorithm affects some value of $\alpha \in[0,100]$ corresponding to fluid domain and so impacts on the size of fluid regions relatively to the solid regions in the domain. 


\subsubsection{Influence of $\tau$.}

It can clearly be seen from Figures 7 and 10 that different designs are obtained for the different values of $\tau$. The parameter $\tau$ in the interpolation functions $h_{\tau}(\alpha)$ and $k_{\tau}$ indicates the stiffness of the curve of the functions. It does not seem to impact significantly the optimization results. However, we can observe in Figure 11 that the frontier between the fluid and the solid is sharper when $\tau=2.0$. In Figure 8, for $\tau=0.3, \tau=0.7$, we can notice residual material in fluid domain while they do not exist anymore when $\tau=2.0$. As the same, the porous region identified in the bend pipe case between the main flow and the hot plate is defined mostly with the fluid diffusivity by the interpolation function. This impact does not influence significantly the result of the optimization computation when we compared their value in Figure 11 but it improves the thermal transition between fluid and solid regions. So, parameter $\tau$ seems to improve the thermal conductivity distribution during the process optimization.

\section{Conclusion}

This study shows that the new interpolation technique based on sigmoid function is a viable method. Design material is well interpolated since intermediate regions between solid and fluid are suppressed hence giving a frontier between the two regions which is well-defined. Besides, the size of transition zones between fluid and solid regions is explicitly computed. The result is that the new interpolation functions reduce their size comparatively to the RAMP interpolation function. Therefore, the transition between fluid and solid is improving thanks to this sigmoid function for the effective conduc- 

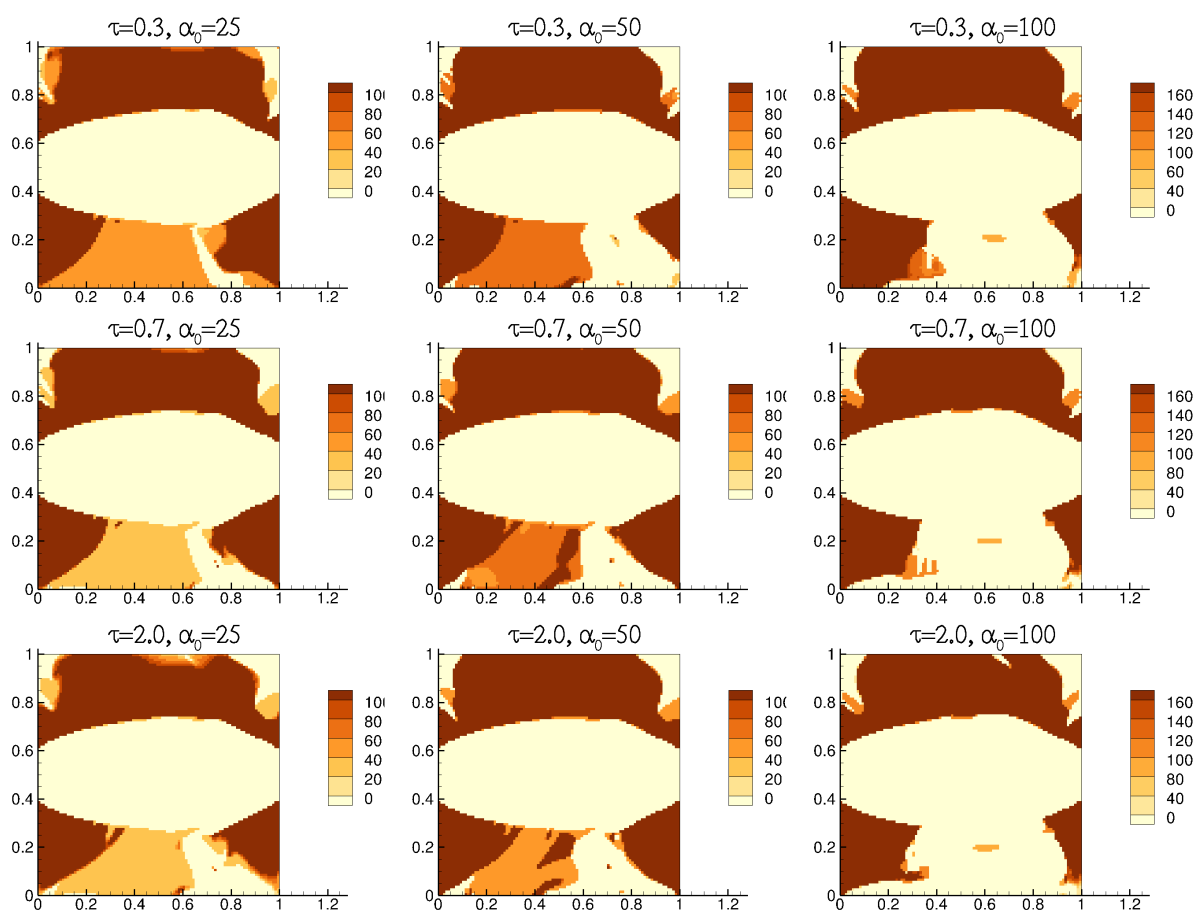

Figure 6: $\alpha$ distribution in the single pipe 

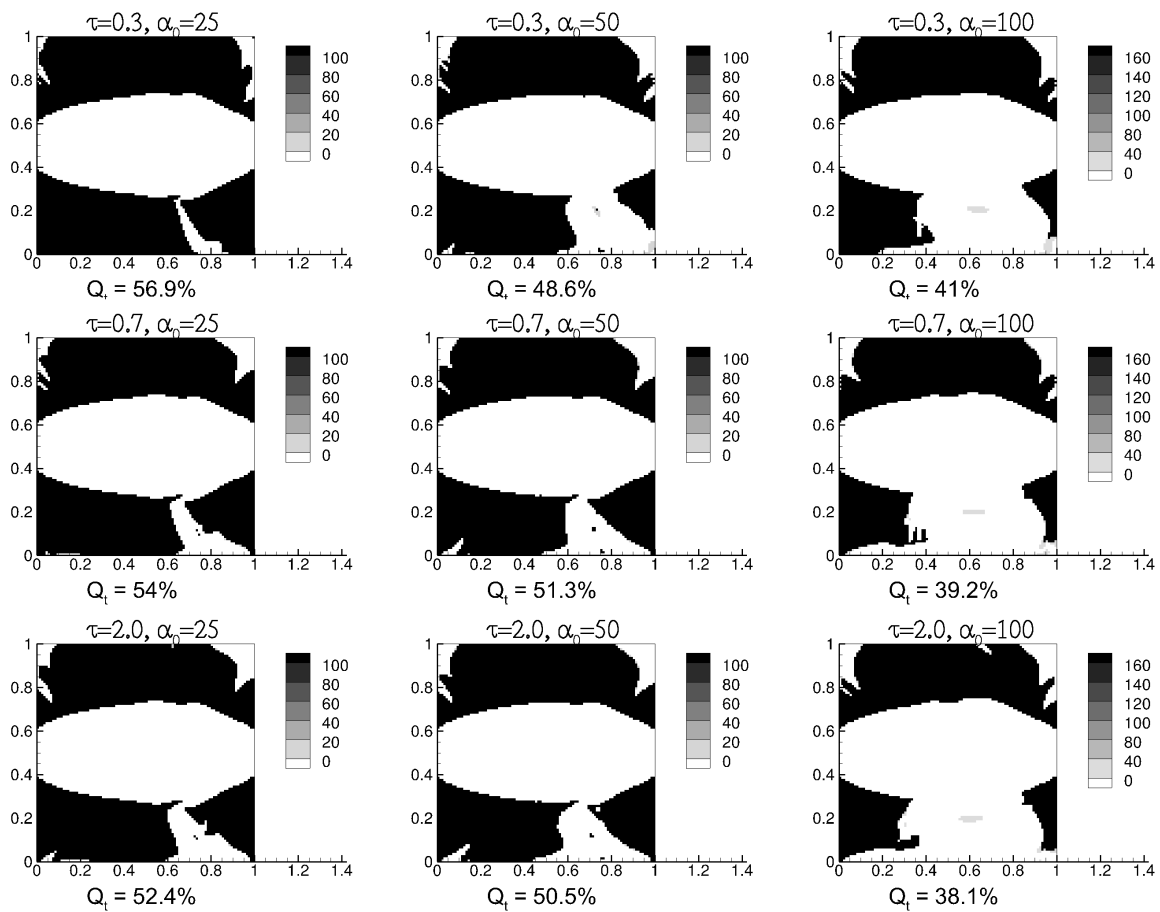

Figure 7: $h_{\tau}(\alpha)$ distribution in the single pipe and proportion of material $Q_{t}$ added in the domain after optimization 

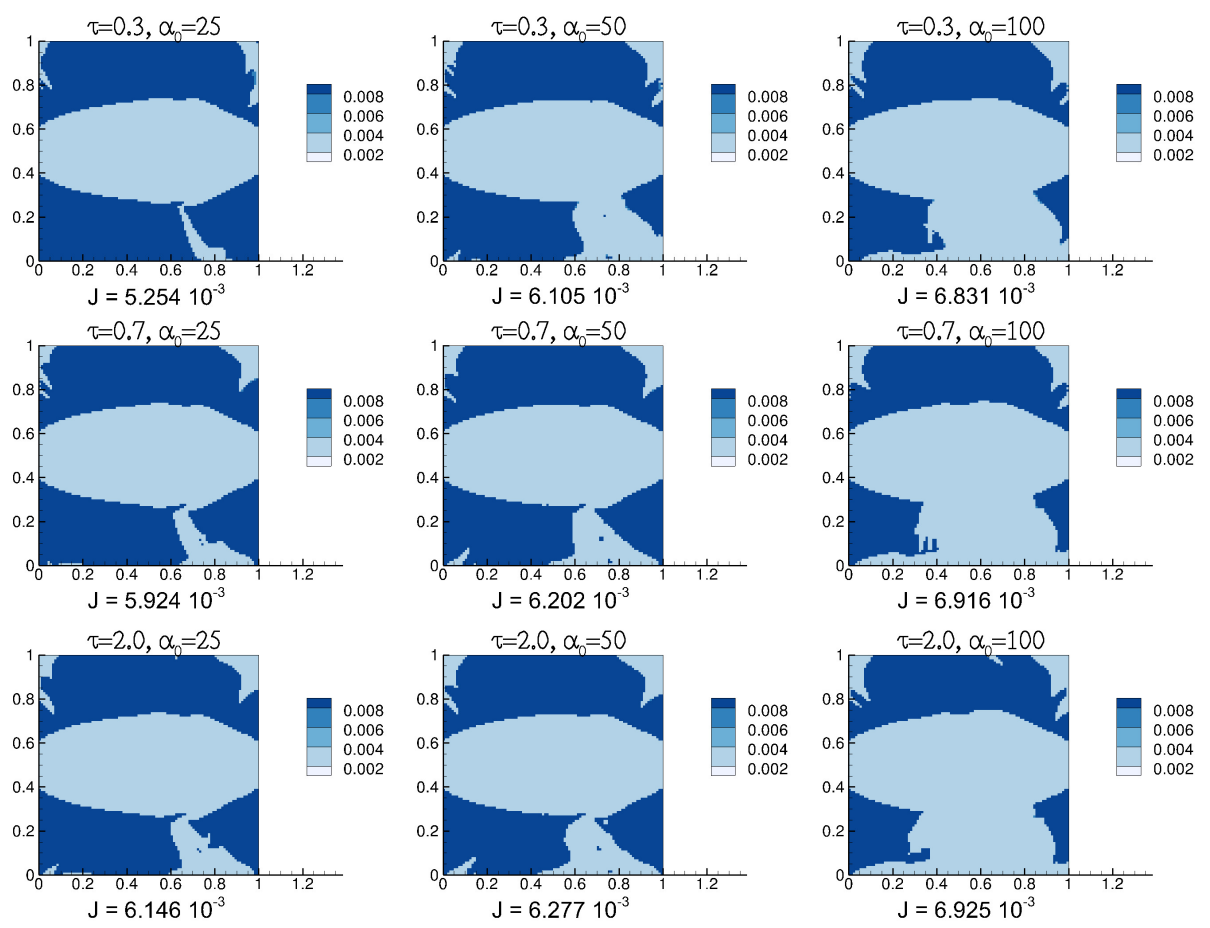

Figure 8: $k_{\tau}(\alpha)$ distribution in the single pipe for different values of $\tau$ and $\alpha_{0}$ and value of functional objectif $\mathcal{J}$ after optimization 


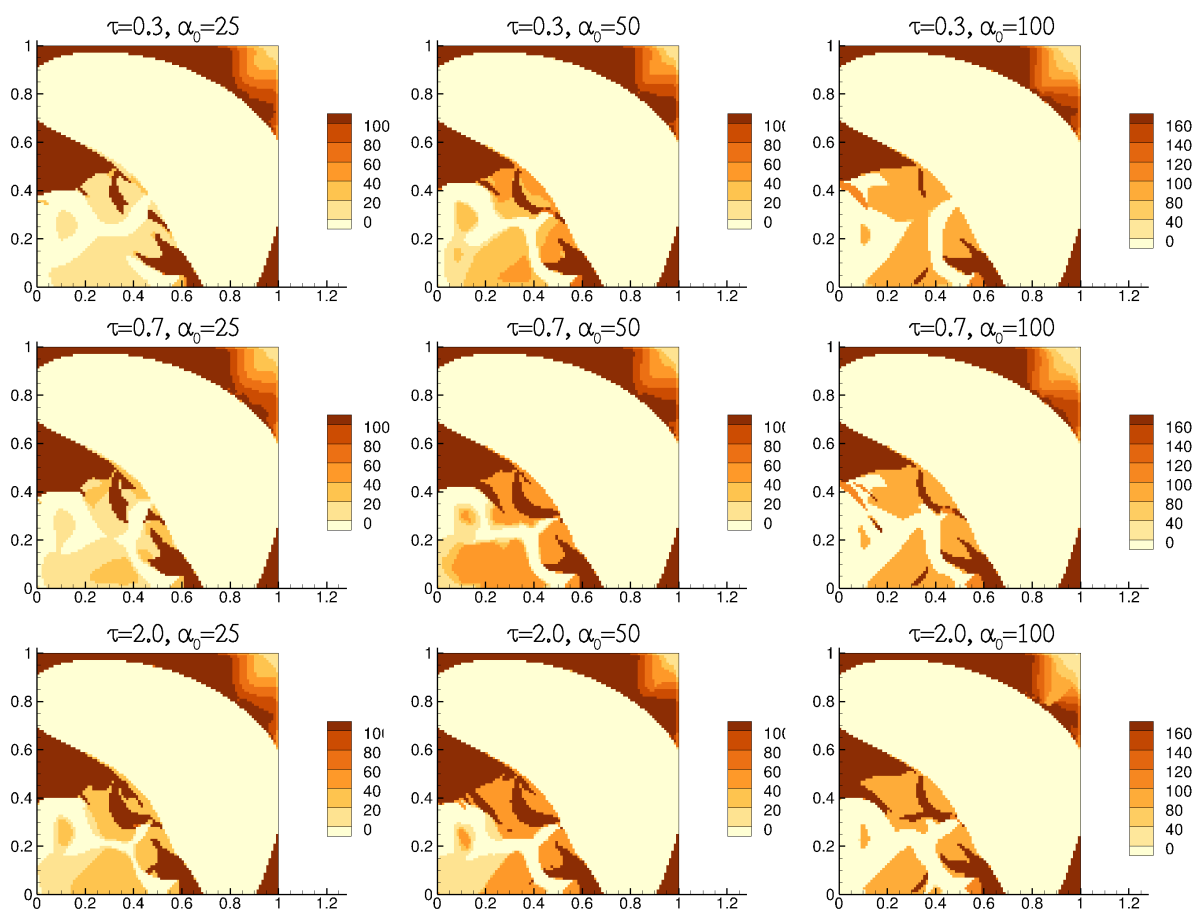

Figure 9: $\alpha$ distribution in the bend pipe 

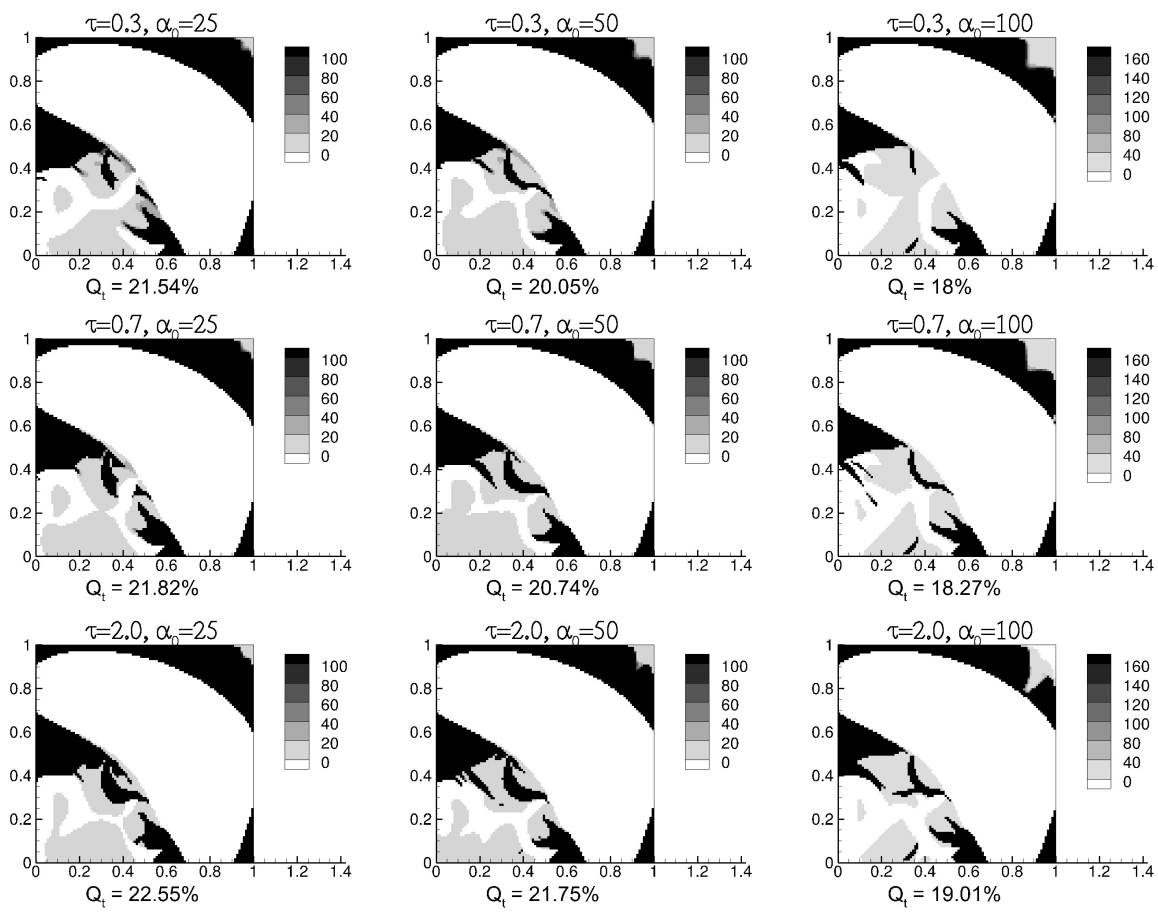

Figure 10: $h_{\tau}(\alpha)$ distribution in the bend pipe and proportion of material $Q_{t}$ added in the domain after optimization 

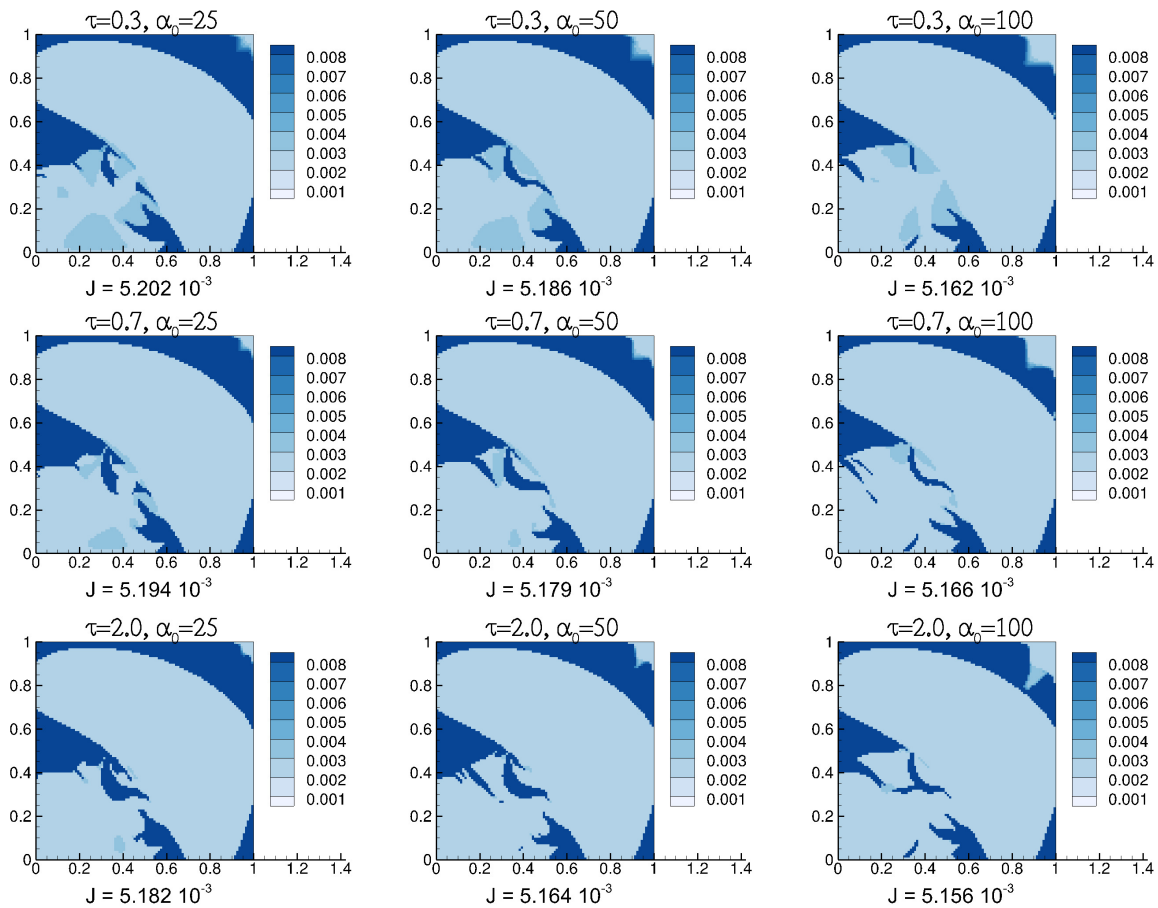

Figure 11: $k_{\tau}(\alpha)$ distribution in the bend pipe and value of functional objectif $\mathcal{J}$ after optimization 
tivity. Computation of the size of transition zones could also be applied to other interpolation functions in order to evaluate the well-definition of the frontier between fluid and solid regions. This new interpolation technique do not require any regularization techniques since no checkerboards have been identified for the two studied cases. Two new parameters have been introduced : the abscissa slope of the sigmoid function $\alpha_{0}$ and the curve of the sigmoid function $\tau$. Their impact has been also investigated. $\alpha_{0}$ has a significant impact on the quantity of material added in the domain during the optimization process. The larger $\alpha_{0}$ is the bigger will be the fluid domain. The parameter $\tau$ has a small impact on the results of optimization. For the studied cases, the impact was seen after interpolation of thermal conductivity. Elements of porous domain have been affected to fluid diffusivity or solid conductivity, and therefore, the thermal transition between material is improved. So, this study has examined a new interpolation technique to deal with fluid-porous media interfaces for topology optimization of heat transfer problems. Further research might explore several type of flow regimes in optimization problems using these interpolation functions. Another possible area of future research would be to investigate new cost functions.

\section{References}

[1] Bends $\phi$ e, Martin Philip, and Ole Sigmund. Topology optimization: theory, methods, and applications. Springer Science \& Business Media, 2013.

[2] Guest, James K., and Jean H. Prvost. "Topology optimization of creep- 
ing fluid flows using a DarcyStokes finite element." International Journal for Numerical Methods in Engineering 66.3 (2006): 461-484.

[3] Kreissl, Sebastian, Georg Pingen, and Kurt Maute. "Topology optimization for unsteady flow." International Journal for Numerical Methods in Engineering 87.13 (2011): 1229-1253.

[4] Borrvall, Thomas, and Joakim Petersson. "Topology optimization of fluids in Stokes flow." International journal for numerical methods in fluids 41.1 (2003): 77-107.

[5] Kreissl, Sebastian, and Kurt Maute. "Levelset based fluid topology optimization using the extended finite element method." Structural and Multidisciplinary Optimization 46.3 (2012): 311-326.

[6] Rozvany, George IN, Ming Zhou, and Torben Birker. "Generalized shape optimization without homogenization." Structural and Multidisciplinary Optimization 4.3 (1992): 250-252.

[7] Lee, Kyungjun, PhD thesis, University of Michigan, Topology Optimization of Convective Cooling System Designs, 2012

[8] Olesen, Laurits H., Fridolin Okkels, and Henrik Bruus. "A highlevel programming-language implementation of topology optimization applied to steady-state Navier-Stokes flow." arXiv preprint physics/0410086 (2004).

[9] Gersborg-Hansen, Allan, Ole Sigmund, and Robert B. Haber. "Topology optimization of channel flow problems." Structural and Multidisciplinary Optimization 30.3 (2005): 181-192. 
[10] Gersborg, Allan Roulund, and Casper Schousboe Andreasen. "An explicit parameterization for casting constraints in gradient driven topology optimization." Structural and Multidisciplinary Optimization 44.6 (2011): 875-881.

[11] Kangro, Urve, and Roy Nicolaides. "Divergence boundary conditions for vector Helmholtz equations with divergence constraints." ESAIM: Mathematical Modelling and Numerical Analysis 33.3 (1999): 479-492.

[12] Petra, Noei, and Georg Stadler. Model variational inverse problems governed by partial differential equations. No. ICES-11-05. Texas univ. at Austin inst. for computational engineering and sciences, 2011

[13] van Dijk, Nico P., et al. "Level-set methods for structural topology optimization: a review." Structural and Multidisciplinary Optimization 48.3 (2013): 437-472.

[14] Evgrafov, Anton. "The limits of porous materials in the topology optimization of Stokes flows." Applied mathematics \& optimization 52.3 (2005): 263-277.

[15] Marck, Gilles, Maroun Nemer, and Jean-Luc Harion. "Topology optimization of heat and mass transfer problems: laminar flow." Numerical Heat Transfer, Part B: Fundamentals 63.6 (2013): 508-539.

[16] Stolpe, Mathias, and Krister Svanberg. "An alternative interpolation scheme for minimum compliance topology optimization." Structural and Multidisciplinary Optimization 22.2 (2001): 116-124. 
[17] Qian, Xiaoping, and Ercan M. Dede. "Topology optimization of a coupled thermal-fluid system under a tangential thermal gradient constraint." Structural and Multidisciplinary Optimization 54.3 (2016): $531-551$.

[18] Othmer, C. "A continuous adjoint formulation for the computation of topological and surface sensitivities of ducted flows." International Journal for Numerical Methods in Fluids 58.8 (2008): 861-877.

[19] Matsumori, T., A. Kawamoto, and T. Kondoh. "Topology optimization for fluidthermal interaction problems." 6th ChinaJapanKorea joint symposium on optimization of structural and mechanical systems. 2010.

[20] Dede, Ercan M. "Multiphysics optimization, synthesis, and application of jet impingement target surfaces." Thermal and Thermomechanical Phenomena in Electronic Systems (ITherm), 2010 12th IEEE Intersociety Conference on. IEEE, 2010.

[21] Dede, Ercan M. "Multiphysics topology optimization of heat transfer and fluid flow systems." proceedings of the COMSOL Users Conference. 2009.

[22] Amir, Oded, and Ole Sigmund. "On reducing computational effort in topology optimization: how far can we go?." Structural and Multidisciplinary Optimization 44.1 (2011): 25-29.

[23] Kim, Yoon Young, and Gil Ho Yoon. "Multi-resolution multi-scale topology optimizationa new paradigm." International Journal of Solids and Structures 37.39 (2000): 5529-5559. 
[24] Herzog, Roland, and Karl Kunisch. "Algorithms for PDEconstrained optimization." GAMMMitteilungen 33.2 (2010): 163-176.

[25] Gunzburger, Max D. Perspectives in flow control and optimization. Society for industrial and applied mathematics, 2002.

[26] Guest, James K., and Lindsey C. Smith Genut. "Reducing dimensionality in topology optimization using adaptive design variable fields." International journal for numerical methods in engineering 81.8 (2010): 1019-1045.

[27] Othmer, Carsten. "Adjoint methods for car aerodynamics." Journal of Mathematics in Industry 4.1 (2014): 1-23.

[28] Andreasen, Casper Schousboe, Allan Roulund Gersborg, and Ole Sigmund. "Topology optimization of microfluidic mixers." International Journal for Numerical Methods in Fluids 61.5 (2009): 498-513.

[29] Alexandersen, Joe, et al. Topology optimisation for coupled convection problems. DTU Mechanical Engineering, 2013.

[30] Alexandersen, Joe, et al. "Topology optimisation for natural convection problems." International Journal for Numerical Methods in Fluids 76.10 (2014): 699-721.

[31] Andreasen, Casper Schousboe, and Ole Sigmund. "Saturated poroelastic actuators generated by topology optimization." Structural and Multidisciplinary Optimization 43.5 (2011): 693-706. 
[32] Okkels, Fridolin, and Henrik Bruus. "Scaling behavior of optimally structured catalytic microfluidic reactors." Physical Review E 75.1 (2007): 016301 .

[33] Deng, Yongbo, et al. "Topology optimization of unsteady incompressible NavierStokes flows." Journal of Computational Physics 230.17 (2011): 6688-6708.

[34] Challis, Vivien J., and James K. Guest. "Level set topology optimization of fluids in Stokes flow." International journal for numerical methods in engineering 79.10 (2009): 1284-1308.

[35] Yoon, Gil Ho. "Topology optimization for stationary fluidstructure interaction problems using a new monolithic formulation." International journal for numerical methods in engineering 82.5 (2010): 591-616.

[36] Yoon, Gil Ho. "Topology optimization for stationary fluidstructure interaction problems using a new monolithic formulation." International journal for numerical methods in engineering 82.5 (2010): 591-616.

[37] Deng, Yongbo, Zhenyu Liu, and Yihui Wu. "Topology optimization of steady and unsteady incompressible NavierStokes flows driven by body forces." Structural and Multidisciplinary Optimization 47.4 (2013): 555570.

[38] Ramalingom D., Cocquet P.-H. \& Bastide A. "Optimisation topologique des échanges thermiques dans un canal vertical asymétriquement chauffé", Accepted for publication in proceeding of the 25th Congrès Français de Thermique (2017) 
[39] Ramalingom D., Cocquet P.-H. \& Bastide A. , Numerical study of natural convection in asymmetrically heated channel considering thermal stratification and surface radiation, Submitted. (2017)

[40] Jenkins, Nicholas, and Kurt Maute. "An immersed boundary approach for shape and topology optimization of stationary fluid-structure interaction problems." Structural and Multidisciplinary Optimization 54.5 (2016): 1191-1208.

[41] Kubo, Seiji, et al. "A level set-based topology optimization method for optimal manifold designs with flow uniformity in plate-type microchannel reactors." Structural and Multidisciplinary Optimization 55.4 (2017): 1311-1327.

[42] Desrayaud, G., et al. "Benchmark solutions for natural convection flows in vertical channels submitted to different open boundary conditions." International Journal of Thermal Sciences 72 (2013): 18-33.

[43] Stolpe, Mathias, and Krister Svanberg. "An alternative interpolation scheme for minimum compliance topology optimization." Structural and Multidisciplinary Optimization 22.2 (2001): 116-124.

[44] Weller, Henry G., et al. "A tensorial approach to computational continuum mechanics using object-oriented techniques." Computers in physics 12.6 (1998): 620-631.

[45] Hinze, Michael, et al. Optimization with PDE constraints. Vol. 23. Springer Science \& Business Media, 2008. 
654

655

656

657

658

659

660

661

662

[46] Zhou, Shiwei, and Qing Li. "A variational level set method for the topology optimization of steady-state NavierStokes flow." Journal of Computational Physics 227.24 (2008): 10178-10195.

[47] Nrgaard, Sebastian, Ole Sigmund, and Boyan Lazarov. "Topology optimization of unsteady flow problems using the lattice Boltzmann method." Journal of Computational Physics 307 (2016): 291-307.

[48] Koga, Adriano A., et al. "Development of heat sink device by using topology optimization." International Journal of Heat and Mass Transfer 64 (2013): 759-772. 